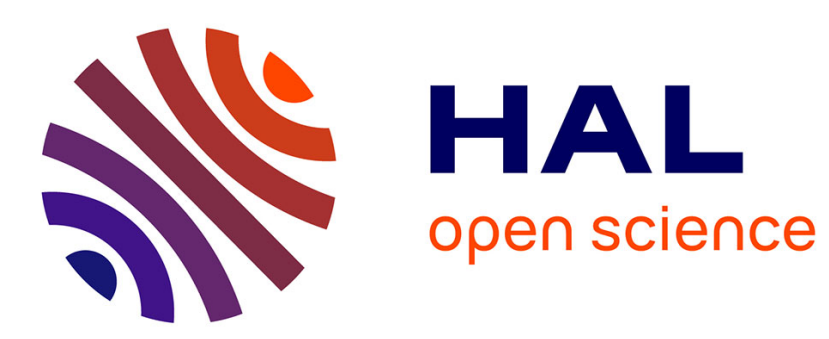

\title{
Complex spectral dimensionality on fractal structures
}

D. Bessis, J.S. Geronimo, P. Moussa

\section{To cite this version:}

D. Bessis, J.S. Geronimo, P. Moussa. Complex spectral dimensionality on fractal structures. Journal de Physique Lettres, 1983, 44 (24), pp.977-982. 10.1051/jphyslet:019830044024097700 . jpa00232297

\section{HAL Id: jpa-00232297 https://hal.science/jpa-00232297}

Submitted on 1 Jan 1983

HAL is a multi-disciplinary open access archive for the deposit and dissemination of scientific research documents, whether they are published or not. The documents may come from teaching and research institutions in France or abroad, or from public or private research centers.
L'archive ouverte pluridisciplinaire HAL, est destinée au dépôt et à la diffusion de documents scientifiques de niveau recherche, publiés ou non, émanant des établissements d'enseignement et de recherche français ou étrangers, des laboratoires publics ou privés. 
Classification

Physics Abstracts

$63.10-63.50-71.10$

\title{
Complex spectral dimensionality on fractal structures
}

\author{
D. Bessis, J. S. Geronimo (*) and P. Moussa \\ Service de Physique Théorique, Centre d'Etudes Nucléaires de Saclay, \\ 91191 Gif sur Yvette Cedex, France
}

(Reçu le 19 octobre 1983, accepté le 2 novembre 1983)

\begin{abstract}
Résumé. - Dans de nombreux problèmes physiques, on associe les propriétés d'invariance d'échelle à l'apparition de structures fractales. Dans le cas d'un modèle soluble, on montre que l'analogie précédente conduit à considérer des indices complexes, par exemple dans les lois de puissance représentant la densité d'état. Les oscillations correspondantes sont nécessaires pour reproduire la partie singulière du spectre, et sont gouvernées par les singularités les plus proches de la transformée de Mellin de la densité d'état. L'observation de telles oscillations dans des systèmes réels pourrait permettre de déterminer la dimension fractale effective des structures impliquées.
\end{abstract}

\begin{abstract}
Fractal structures have been associated with scaling properties of many physical systems. On the basis of a solvable model, it is asserted that the above analogy leads in a natural way to complex indices, for instance, in the power law describing the density of states. The corresponding oscillations are, in fact, necessary to reproduce the singular part of the spectrum, and are governed by the nearest complex singularities of the Mellin transform of the spectral density. Observation of such oscillations in actual systems might allow the effective fractal dimension of the involved structure to be determined.
\end{abstract}

In many physical problems where scaling properties play an important rôle, a detailed interpretation of the observed phenomena has been proposed [1,2] in terms of fractal structures. This is the case for the vibration properties of proteins [3], percolation in discontinuous thin films [4], diamagnetic properties of superconductors near the percolation threshold [5]. In fact, fractal structures seem to generate in a natural way singular spectral measures [6] for the vibration spectrum, or singular continuous density of zeroes in statistical models on fractal hierarchical lattices [7].

On the other hand, a singular part arises in the spectrum in some cases for the Schrödinger equation with an almost periodic potential [8]. Such equations have been considered for incommensurate structures, conducting or super-conducting linear chains [9], electronic properties of crystals in a magnetic field [10], and more generally in the metal-insulator transition. It is a somewhat surprising fact that almost periodicity and renormalization group properties may be related, and there exist exact models sharing both properties [11].

$\left({ }^{*}\right)$ Permanent address : School of Mathematics, Georgia Institute of Technology, Atlanta, Georgia 30332, U.S.A., supported in part by a N.A.T.O. Postdoctoral fellowship. 
An important feature of the density of state associated with a Schrödinger Hamiltonian is its behaviour for low energy excitations. Near the edge of the spectrum $E_{\text {end }}$, the density of states behaves as

$$
\rho(E) \sim C\left(E-E_{\text {end }}\right)^{(\delta / 2)-1}
$$

where $\delta$ is the spectral dimension of the system $[12,5]$. The spectral dimension $\delta$ is usually different from the geometrical fractal dimension of the system [1].

The purpose of this letter is to show that, in the case of a singular spectrum, one should expect that the constant $C$ has to be replaced by an oscillating term :

$$
\rho(E) \sim\left(E-E_{\text {end }}\right)^{(\delta / 2)-1}\left\{\bar{C}+\sum_{k} a_{k} \cos \left(k \tau \ln \left(E-E_{\text {end }}\right)+\varphi_{k}\right)\right\} .
$$

The " constant " $C$ in (1) has in fact no definite limit. A simple intuitive argument follows from the fact that a purely singular continuous spectrum has infinitely many holes near its edge, and the oscillations are required to reproduce this feature. The same argument holds for a pure point spectrum with support in a Cantor-like set [6].

In order to be more precise, we shall briefly explain the situation in a particular model : the quadratic mapping Hamiltonian [11], which has the significant advantage of being solvable. The model is one dimensional, discrete and semi-infinite with wave function $\psi_{n}$ defined for integer $n \geqslant 0$. The Hamiltonian satisfies a renormalization group equation

$$
H D=D\left(H^{2}-\lambda\right), \quad \lambda \geqslant 2
$$

where $D$ is a decimation operator :

$$
(D \psi)_{n}=\psi_{2 n}
$$

The spectrum of $H$ is invariant under the transformation

$$
T(E)=E^{2}-\lambda,
$$

since $D \psi$ is a (quasi) eigenstate with eigenvalue $E^{2}-\lambda$ when $\psi$ is any (quasi) eigenstate with eigenvalue $E$.

We recall some properties of the model $[11,13]$ :

i) The spectrum is purely singular, its support reduces to a Cantor set of Lebesgue measure zero, which consists of all points of the form :

$$
\pm \sqrt{\lambda \pm \sqrt{\lambda \pm \sqrt{\lambda \ldots}}}
$$

which is nothing but the Julia set $[14,15] J$ associated to (5).

ii) The wave functions are the set of polynomials $P_{n}(E)$ orthogonal with respect to the invariant measure naturally associated with the set [14].

iii) The resolvant in the state $\psi^{(0)}$ defined by :

$$
\left(\psi^{(0)}\right)_{n}=\delta_{0, n}
$$

is the generating function associated to the density of state $\mu(E)$, integrated on the set $J$ :

$$
g(z)=\left\langle\psi^{(0)}\left|(1-z H)^{-1}\right| \psi^{(0)}\right\rangle=\int_{J} \frac{\mathrm{d} \mu(E)}{1-z E} .
$$

iv) The eigenstates have chaotic behaviour [11] which will not be discussed here. 
v) The end point of the spectrum is here defined as :

$$
E_{\text {end }}=a(\lambda)=1 / 2+(\lambda+1 / 4)^{1 / 2},
$$

which is the largest fixed point of the spectrum. Here for the sake of convenience we will analyse the density of states near the maximum eigenvalue. An obvious change of sign in the energy variable will make the link with the usual convention.

The "Lyapunov function " which expresses the large $n$ behaviour of the eigenstates [16] is given by

$$
G(z)=\int_{-a}^{+a} \ln (z-E) \mathrm{d} \mu(E)
$$

and is related to the resolvant $g(z)$ by :

$$
g(z)=\frac{1}{z} G^{\prime}(1 / z) .
$$

We have the functional equation

$$
G(z)=\frac{1}{2} G\left(z^{2}-\lambda\right)
$$

First we note that the general solution $G(z)$ of $(12)$ is related to a particular solution $G_{0}(z)$ in terms of a periodic function $p(x)$ :

$$
p(x)=p(x+\ln 2)
$$

through

$$
G(z)=G_{0}(z) p\left(\ln G_{0}(z)\right)
$$

Using the Fourier expansion of (13) :

$$
p(x)=\sum_{-\infty}^{+\infty} p_{m} \exp (2 i \pi m x / \ln 2)
$$

we get a formal expansion of the general solution of (12)

$$
G(z)=\sum_{-\infty}^{+\infty} p_{m}\left(G_{0}(z)\right)^{1+\frac{2 i \pi m}{\ln 2}}
$$

It is convenient to choose $G_{0}(z)$ as the solution which admits an expansion around a :

$$
G_{0}(z)=\left(\frac{z-a}{2 a}\right)^{\frac{\ln 2}{\ln 2 a}} \sum_{n=0}^{\infty} g_{n, 0}\left(\frac{z-a}{2 a}\right)^{n} .
$$

Introducing the elementary solution

$$
G_{m}(z)=\left[G_{0}(z)\right]^{1+\frac{2 i \pi m}{\ln 2}}
$$


we get :

$$
G(z)=\sum_{-\infty}^{+\infty} p_{m} G_{m}(z)=\sum_{m} \sum_{n} p_{m} g_{n, m}\left(\frac{z-a}{2 a}\right)^{\frac{\ln 2}{\ln 2 a}+n+\frac{2 i \pi m}{\ln 2 a}}
$$

Noting that $G(z)$ is real analytic, we get :

$$
G(z)=\sum_{m=0}^{\infty} \sum_{n=0}^{\infty} \rho_{n, m}\left(\frac{z-a}{2 a}\right)^{\frac{\ln 2}{\ln 2 a}+n}\left\{\cos \left(\frac{2 \pi m}{\ln 2} \ln \left(\frac{z-a}{2 a}\right)+\varphi_{m, n}\right)\right\}
$$

where the $\rho_{n, m}$ and $\varphi_{m}$ are real parameters. This expression is an asymptotic expansion for the Green function and a related expansion can be obtained for the density of states :

$$
\rho(E)=\sum_{m=-\infty}^{m=+\infty} \sum_{n=0}^{\infty} \delta_{m, n}\left(\frac{E_{\mathrm{end}}-E}{2 E_{\mathrm{end}}}\right)^{\frac{\ln 2}{\ln \left(2 E_{\mathrm{end}}\right)}+n+\frac{2 i \pi m}{\ln \left(2 E_{\mathrm{end}}\right)}-1}
$$

which leads to (2) in view of the reality conditions. A precise mathematical statement is expressed in terms of the Mellin transform :

$$
M(s)=\int_{J}\left(E_{\text {end }}-E\right)^{s} \rho(E) \mathrm{d} E
$$

which can be proven to be a meromorphic function [17] with poles on a semi infinite lattice, at positions :

$$
\left.\begin{array}{l}
s_{m, n}=-2 \delta-n+i m \tau, \quad n \in \mathbb{N}, \quad m \in \mathbb{Z} \\
\tau=\frac{2 \pi}{\ln \left(2 E_{\text {end }}\right)} \quad \frac{\delta}{2}=\frac{\ln 2}{\ln \left(2 E_{\text {end }}\right)} .
\end{array}\right\}
$$

The dominant part in the oscillations is provided by poles $s_{0, \pm 1}$. The proof of the meromorphy follows from the invariance of the spectral measure under the transformation (5). Note also that $M(s)$ can be continued analytically toward real negative integers and one gets [17]

$$
M(-n)=0 \text {. }
$$

This relation (commonly named trace identity) may provide useful sum rules for the spectral density.

A generalization to higher degree polynomial transformation will provide similar results [18]. Although the amplitude of the oscillations may be rather small (a few per cent in our case for $\lambda=3$ ), and can only be evaluated numerically, we think the phenomenon is quite generally related to functional equations like (12), where the transformation is an analytic function as is $T$ in (5). Such oscillations occur in real variable problems [19] for instance in the behaviour of infinite products of random matrices [20], where the Lyapunov index may display a behaviour similar to (2).

In statistical mechanics, critical complex indices have already been considered in the renormalization group analysis [21] but they are usually rejected for translation invariant systems since they imply a length scale, or a finite size. However they appear when an exact renormalization group transformation occurs, as is the case for Ising or Potts models on hierarchical lattices [7]. In this case the fractal structure occurs for the density of zeroes itself, generating an oscillatory critical behaviour of the thermodynamical quantities as a function of the temperature, superimposed on the usual scaling laws. 
We shall now discuss in more detail the relevance of our discussion to fractal structures. A fractal object has a discrete scaling semi group, with scale parameter $b$, the number of degrees of freedom being concomitantly rescaled as $b^{d}$, where $\bar{d}$ is the relevant fractal dimension. The usual scaling arguments applied to the density of states give the following relation $[2,6]$ near the low frequency fixed point :

$$
\rho\left(E-E_{\text {end }}\right) \simeq \frac{1}{b^{d}} \rho\left(\mu\left(E-E_{\text {end }}\right)\right)
$$

where $\mu$ is the renormalization group eigenvalue. From the latter equation we get [19]

$$
\left.\begin{array}{c}
\rho\left(E-E_{\text {end }}\right) \sim\left(E-E_{\text {end }}\right)^{(\delta / 2)-1} f\left(2 \pi \tau\left(E-E_{\text {end }}\right)\right) \\
\frac{\delta}{2}=1+\bar{d} \frac{\ln b}{\ln \mu} \\
\tau=\frac{1}{\ln \mu}
\end{array}\right\}
$$

where :

and $f$ is a periodic function with period 1 .

Therefore the low frequency behaviour of the density of states is governed by two numbers $\delta$ and $\tau$, the knowledge of which allows one to determine the scaling factor $b^{d}$ of the number of degrees of freedom, as well as the fractal dimension $\bar{d}$. In our case (Eq. 23 above), $b=2, \bar{d}=1$, $\mu=2 a$. Preliminary numerical calculations indicate that the Fourier coefficients of the function $f$ in (26) decrease very rapidly, so that $f(t)$ is expected to be well reproduced by $C_{0}+C_{1} \cos (2 \pi t)$.

To summarize, we think that the oscillations of the density of states, as well as the corresponding critical oscillations in statistical mechanics, may lead to observable phenomena in physical systems which possess a tendency to generate a singular measure associated in a natural way to a renormalization group transformation. This singular measure may be either singular continuous or pure point, but in both cases the essential spectrum is a Cantor set. The models considered may include both almost periodic and fractal structures. From a practical point of view, the observation of such oscillations in actual systems would also provide an estimation of the effective fractal dimensionality of the involved structure.

\section{References}

[1] Gefen, Y., Aharony, A., Mandelbrot, B., KirkPatrick, S., Phys. Rev. Lett. 47 (1981) 1771.

[2] Alexander, S., Orbach, R., J. Physique Lett. 43 (1982) L-625.

[3] Stapleton, H. J., Allen, J. P., Flynn, C. P., Stinton, D. G., Kurtz, S. R., Phys. Rev. Lett. 45 (1980) 1456.

[4] Voss, R. F., Laibowitz, R. B., Allessandrini, E. I., Phys. Rev. Lett. 49 (1982) 1441 ; Kapitulnik, A., Deutscher, G., Phys. Rev. Lett. 49 (1982) 1444.

[5] Rammal, R., Lubensky, T. C., Toulouse, G., J. Physique-Lett. 44 (1983) L-65.

[6] Domany, E., Alexander, S., Bensimon, D. and Kadanoff, L. P., Phys. Rev. B 28 (1983) 3110.

Rammal, R., Spectrum of harmonic excitations on fractals, to appear in J. Physique (Paris).

Rammal, R., Toulouse, G., Phys. Rev. Lett. 49 (1982) 1194.

Langlois, J. M., Tremblay, A. M. S., Southern, B. W., Phys. Rev. B 28 (1983) 218.

[7] Derrida, B., DE Seze, L., ITzykson, C., Fractal structures of zeroes in hierarchical models, Saclay DPhT preprint (1983), and references therein, to appear in J. Stat. Phys. 
[8] Hofstadter, D. R., Phys. Rev. B 14 (1967) 2239.

Aubry, S., André, G., Ann. Israel Phys. Soc. 3 (1980) 111.

Avron, J. and Simon, B., Bull. Amer. Math. Soc. 6 (1982) 81.

[9] Turkevitch, L. A., Klemm, R. A., Phys. Rev. B 19 (1979) 2520.

[10] Harper, P. G., Proc. Phys. Soc. A 68 (1980) 874.

Azbel, M. Ya., Sov. Phys. JETP 19 (1964) 634.

[11] Bellissard, J., Bessis, D., Moussa, P., Phys. Rev. Lett. 49 (1982) 701.

[12] Dhar, D., J. Math. Phys. 18 (1977) 577.

[13] Bessis, D., Mehta, M. L., Moussa, P., Letters Math. Phys. 6 (1982) 123.

[14] For a review see Brolin, H., Arkiv. Mat. 6 (1965) 103.

[15] Barnsley, M. F., Geronimo, J. S., Harrington, A. N., Commun. Math. Phys. 88 (1983) 479.

[16] Thouless, D. J., J. Phys. C 5 (1972) 77.

[17] Bessis, D., Geronimo, J. S., Moussa, P., Mellin transforms associated with Julia sets and physical applications, Saclay DPhT Preprint (1983) to appear in J. Stat. Phys.

[18] Barnsley, M. F., Geronimo, J. S., Harrington, A., Bull. Am. Math. Soc. 7 (1982) 381.

Bessis, D., Moussa, P., Commun. Math. Phys. 88 (1983) 503.

[19] Derrida, B., Eckmann, J. P. and Erzan, A., J. Phys. A 16 (1983) 893.

[20] Derrida, B. and Hilhorst, H. J., J. Phys. A 16 (1983) 2641.

[21] NiemeiJer, Th., Van Leeuwen, J. M. J., Renormalization Theory for Ising like spin systems, Vol. 6, in Phase Transition and critical phenomena, C. Domb and M. S. Green Editors (Academic Press) 1976, p. 425. 